\title{
Comparison of the Sanofi Diagnostics Pasteur Chlamydia Microplate EIA shortened assay with the original standard assay and cell culture
}

\author{
E. L. CHAN, K. BRANDT, HEATHER STONEHAM and G. HORSMAN \\ Virology Section, Department of Clinical Microbiology, Provincial Laboratory, Regina, Saskatchewan S4S 5 W6, \\ Canada
}

The new Sanofi Diagnostics Pasteur Chlamydia Microplate EIA shortened assay was evaluated by comparison with the original standard assay and cell culture. A total of 853 paired male and female genital tract specimens was tested with both Sanofi Chlamydia Microplate EIA shortened and standard assays and the results were compared with those of cell culture. For confirmation, a blocking assay run in the shortened format was used. Discrepancies between the three methods were resolved by a direct fluorescent antibody (DFA) test on the EIA samples or the culture retentate, or both. After resolution of discrepant results, the standard assay had a sensitivity, specificity, positive predictive value and negative predictive value of $98.5 \%, 100 \%, 100 \%$ and $99.9 \%$, respectively. The shortened assay results were $100 \%, 100 \%, 100 \%$ and $100 \%$, respectively. The shortened assay takes approximately $1.5 \mathrm{~h}$ less time than the standard assay and this study demonstrated that they have equivalent sensitivity and specificity. The improvement in turnaround time enables results to be reported on the same day.

\section{Introduction}

Chlamydia trachomatis infection has been identified as the leading cause of pelvic inflammatory disease (PID), infertility and ectopic pregnancy in women [1]. It is also the commonest cause of sexually transmitted disease [2], with an estimated 4 million infections annually in the USA [3]. Because of the elusive nature of the organism, it is not well recognised and often goes untreated. The estimated direct and indirect cost of chlamydia infections exceed $\$ 2.4$ billion annually in the USA [4]. Accurate diagnosis and treatment of chlamydia infections are of public health importance. Laboratory diagnosis of $C$. trachomatis infection can be performed by cell culture, enzyme immunoassay, direct immunofluorescence assay, DNA hybridisation and nucleic acid amplification techniques. The sensitivity and specificity of each technique varies and DNA amplification techniques probably provide the most sensitive and specific assays $[5,6]$. However, although there have been efforts to make these assays convenient in the clinical laboratory, they are not ideal in a high volume laboratory that tests $>100$ specimens/day.

Received 31 July 1996; revised version accepted 29 Dec. 1996.

Corresponding author: Dr E. L. Chan.
Enzyme immunoassay, on the other hand, offers good sensitivity and specificity as a screening assay [7, 8] and also offers high throughput with rapid turnaround time. The present study was designed to evaluate the new Sanofi Diagnostics Pasteur (SDP) Chlamydia Microplate EIA shortened assay, which is $1.5 \mathrm{~h}$ shorter in turnaround time than the original standard assay. Both EIAs were compared with cell culture in a high volume clinical laboratory. In addition to the comparison of sensitivity and specificity, the difference in turnaround time between the two assays after confirmation was also evaluated to determine whether there is added value in a shorter assay.

\section{Materials and methods}

\section{Patient samples}

Seven hundred and thirty-two endocervical specimens were collected by several family physicians and by staff at the STD Clinic in the General Hospital in Regina. One hundred and twenty-one urethral swabs from male patients were obtained predominantly from the Regina STD clinic. Most of the female patients from the family physician clinics were asymptomatic, while most of the STD clinic patients were either symptomatic or positive contacts. The swab for cell culture 
was collected first in all patients and transported in 2-SP medium in an ice pack. The SDP swab was collected after the cell culture and used in both shortened and standard assays. All specimens were transported to the laboratory within $24 \mathrm{~h}$ of collection.

\section{Culture}

Glass beads were added to the specimens and shaken to break up the cells before inoculation on to a confluent monolayer of McCoy cells previously grown for $24 \mathrm{~h}$ on coverslips in shell vials. The vials were centrifuged at $2500 \mathrm{~g}$ at $35^{\circ} \mathrm{C}$ for $1 \mathrm{~h}$, followed by replacement of the supernate with maintenance medium containing cycloheximide $1 \mu \mathrm{g} / \mathrm{mL}$. The medium was removed after incubation for $72 \mathrm{~h}$ at $37^{\circ} \mathrm{C}$. The cells were stained with a FITC-conjugate (Syva, Ontario, Canada) comprised of monoclonal antibodies prepared to chlamydial reticulate and elementary bodies after methanol fixation. The specimens were examined with a UV epi-fluorescence microscope (Olympus, Alberta, Canada) for the presence of inclusion bodies in the cells. Positive and negative controls were included with each run.

\section{EIA assays}

Sanofi Diagnostics Pasteur Chlamydia Microplate EIA shortened and standard protocols were performed according to the manufacturer's instructions. The blocking assay for confirmation was done according to the shortened assay protocol. A $50 \%$ or greater reduction in absorbance in comparison with the corresponding well without blocking reagent designated positive confirmation.

\section{Cytocentrifugation and direct fluorescent-antibody assay}

The cell culture retentate, all EIA positive specimens, and specimens that fell into the negative grey zone range $(30 \%$ below the cutoff) were tested by cytocentrifugation and direct fluorescent antibody (DFA) assay for the presence or absence of elementary bodies. Cell culture retentates were lysed by a freeze and thaw process before the DFA procedure. SDP does not have a grey zone in the manufacturer's recommended procedure and this was created for the purpose of the study to detect false negative results. DFA slides were prepared by centrifugation of the sample $(400 \mu \mathrm{l})$ at $10000 \mathrm{~g}$. The pellets were resuspended in $20 \mu \mathrm{l}$ of double distilled water and $5 \mu \mathrm{l}$ was spotted on to a slide. After fixation with methanol, the slides were stained with a direct antigen stain; Sanofi Diagnostic Pasteur's Kallestad $^{\mathrm{TM}}$ Direct Antigen (Chaska, MN, USA) anti-MOMP and anti-LPS stain were used in this procedure as the Syva anti-LPS stain will not work with EIA extracts.

All slides were examined with a UV epifluorescence microscope. A positive DFA was defined as five or more EBs.

\section{Criteria for resolving discrepant results}

The specimen was considered positive if the cell culture was positive. If the EIA result differed from the culture result, the discrepancy was resolved as follows. Negative grey zone $(30 \%)$ EIA samples were further tested by DFA and blocking assays. If the EIA specimen was confirmed to be positive by the blocking assay but was culture negative, DFA testing of the EIA sample or the culture retentate was done to further evaluate the sample. If any DFA tested sample was positive with five or more EBs, then the sample was considered confirmed positive and used in the resolution calculation.

\section{Statistical analysis}

Sensitivity, specificity and positive and negative predictive values were calculated in direct comparison with culture and after resolution by DFA.

\section{Results and discussion}

The results of the two assays after resolution of discrepancies are shown in Table 1. There were three discrepancies with the shortened assay and four with the standard assay that were culture negative and EIA positive. After resolution, all the culture retentates from these EIA positives were found to have more than five EBs and thus the EIA results were considered true positives. However, one culture positive sample was negative in the standard EIA assay. All these discrepancies were found in specimens from female patients. The sensitivity and specificity (Table 2) were slightly better in the shortened assay, but the difference was not statistically significant $(\mathrm{p}<0.1)$.

Laboratory diagnosis of $C$. trachomatis infection

Table 1. Results of shortened and standard EIA assays and of culture for C. trachomatis after resolution of discrepancies

\begin{tabular}{|c|c|c|c|c|c|c|c|c|}
\hline \multirow{3}{*}{$\begin{array}{l}\text { Characteristics } \\
\text { Culture/EIA } \\
\text { Female }\end{array}$} & \multicolumn{4}{|c|}{ Shortened assay } & \multicolumn{4}{|c|}{ Standard assay } \\
\hline & $+/ t$ & $-/+$ & $+1-$ & $-1-$ & $+/+$ & $-1+$ & $+1-$ & $-1-$ \\
\hline & 56 & 3 & 0 & 673 & 54 & 4 & 1 & 673 \\
\hline Male & 8 & 0 & 0 & 113 & 8 & 0 & 0 & 113 \\
\hline Overall & 64 & $3^{*}$ & 0 & 786 & 62 & $4^{*}$ & 1 & 786 \\
\hline
\end{tabular}

* Culture retenate showed five or more EBs, thus the EIA is a true positive. 
Table 2. Sensitivity, specificity and predictive values of shortened and standard EIA assays for C. trachomatis.

\begin{tabular}{lll}
\hline Criteria & Shortened assay & Standard assay \\
\hline Sensitivity & $100 \%(67 / 67)$ & $98.5 \%(66 / 67)$ \\
Specificity & $100 \%(786 / 786)$ & $100 \%(786 / 786)$ \\
Positive predictive value & $100 \%(67 / 67)$ & $100 \%(66 / 66)$ \\
Negative predictive value & $100 \%(787 / 787)$ & $99.9 \%(786 / 787)$ \\
\hline
\end{tabular}

continues to be both a science and an art. Clinicians require a reliable test with good predictive values [9], while the clinical laboratory would like to have such a test but also requires low costs, ease of performance and the ability to process large numbers of specimens [10]. Also, the turnaround time should be as short as possible. The Sanofi Diagnostics Pasteur Chlamydia Microplate EIA standard assay has the potential to fulfill all of the above criteria. However, it has an assay time of $c .3 .5 \mathrm{~h}$, which together with the blocking assay for confirmation of positive results, means that it could take at least $7 \mathrm{~h}$ before the final result becomes available. This is close to the end of the working day and could delay the treatment plan. The new DNA amplification methods such as PCR and LCR seem to be more sensitive and specific than EIA assays [5]. However, these new assays require considerable time for specimen preparation and a high degree of technical skill and extra care is required to prevent contamination. Commercial companies have developed automated instrumentation and reduced preparation time to make these assays more acceptable to the routine clinical laboratory, but at this time, most clinical laboratories are not doing these assays routinely or in high volumes. EIA is still the only assay that can be easily applied to a routine high volume laboratory with a reasonable turnaround time.

The present study evaluated the new shortened SDP assay in comparison with the original standard assay and with cell culture. The shortened assay appears to be slightly better in sensitivity and specificity (not statistically significant) and gives good correlation with the results of cell culture. The major advantage of the new shortened assay is the reduction of $1.5 \mathrm{~h}$ in processing time. Taking into account the blocking assay to confirm positive results after the initial run, results can be available in $c .3 \mathrm{~h}$. This greatly improves the turnaround time and in our evaluation, the final confirmed result was usually available by noon. With the implementation of electronic reporting, it is possible to have the final results reported by early afternoon, thus allowing the physician time to inform the patient and initiate treatment.
The creation of a grey zone was due to our previous finding that certain EIA tests may give false negative results if they have an absorbance value in the area $30 \%$ below the cut-off [9]. However, in the present study, there were very few specimens that fell into this zone and these were found to be true negatives. Thus a grey zone does not seem necessary for the shortened assay. The SDP shortened assay and the original standard assay were found to be equivalent with good predictive values. The major advantage of the shortened assay is the decreased turnaround time, which is an asset to the laboratory and improves patient care in an important public health disease.

\section{References}

1. Homes KK. The chlamydia epidemic. JAMA 1981; 245: 1718-1723.

2. Schachter J. Epidemiology of Chlamydia trachomatis infections. In: De la Maza, LM, Peterson EM (eds) Medical virology II. New York, Elsevier Biomedical. 1983; 111-120.

3. Washington AE, Johnson RE, Sanders LL. Chlamydia trachomatis infections in the United States. What are they costing us? JAMA 1987; 257: 2070-2072.

4. Washington $\mathrm{AE}, \mathrm{Katz} \mathrm{P}$. Cost of and payment source for pelvic inflammatory disease. Trends and projections, 1983 through 2000. JAMA 1991; 266: 2565-2569.

5. Chernesky MA, Jang D, Lee $\mathrm{H}$ et al. Diagnosis of Chlamydia trachomatis infections in men and women by testing first-void urine by ligase chain reaction. J Clin Microbiol 1994; 32: 2682-2685.

6. Thejls H, Gnarpe J, Gnarpe H et al. Expanded gold standard in the diagnosis of Chlamydia trachomatis in a low prevalence population: Diagnostic efficacy of tissue culture, direct immunofluorescence, enzyme immunoassay, PCR and serology. Genitourin Med 1994; 70: 300-303.

7. Moncada J, Schachter J, Bolan G et al. Evaluation of Syva's enzyme immunoassay for the detection of Chlamydia trachomatis in urogenital specimens. Diag Microbiol Infect Dis 1992; 15: $663-668$.

8. Clark A, Stamm WE, Gaydos C et al. Multicenter evaluation of the AntigEnz Chlamydia enzyme immunoassay for diagnosis of Chlamydia trachomatis genital infection. J Clin Microbiol 1992; 30: $2762-2764$

9. Chan EL, Brandt K, Kozoriz D, Mushanski L, Spence C, Horsman G. The use of a confirmation assay to increase the sensitivity and specificity of the Chlamydiazyme test. Am J Clin Pathol 1994; 102: 724-728.

10. Chan EL, Brandt K, Horsman GB. A 1-year evaluation of Syva Micro Trak Chlamydia enzyme immunoassay with selective confirmation by direct fluorescent-antibody assay in a highvolume laboratory. J Clin Microbiol 1994; 32: 2208-2211. 